\title{
O Problema da Deposição Gamma é NP-Completo
}

\author{
Marcelo Fonseca Faraj $^{1}$, Sebastián Urrutia ${ }^{1}$, João Fernando Machry Sarubbi ${ }^{2}$ \\ ${ }^{1}$ DCC - Universidade Federal de Minas Gerais (UFMG) \\ Belo Horizonte $-\mathrm{MG}-$ Brasil \\ ${ }^{2}$ DECOM - Centro Federal de Educação Tecnológica de Minas Gerais (CEFET-MG) \\ Belo Horizonte - MG - Brasil \\ marcelo@gmail.com, surrutia@dcc.ufmg.br, joao@decom.cefetmg.br
}

\begin{abstract}
Gamma Deployment is a metric to evaluate the quality of service in vehicular ad hoc networks (VANETs). Gamma Deployment Problem consists in applying that metric to minimize the amount of RSUs deployed in a VANET. In this work, we present a proof that the that problem is NP-Complete.
\end{abstract}

Resumo. Deposição Gamma é uma métrica usada para avaliar a qualidade de serviço oferecida por redes veiculares (VANETs). O Problema da Deposição Gamma consiste no emprego dessa métrica na minimização de RSUs para compor VANETs. Neste trabalho, prova-se que esse problema é NP-Completo.

\section{Introdução}

Deposição Gamma $\left(\Gamma_{D}\left(\begin{array}{l}\tau \\ \rho\end{array}\right)\right.$ ) (SILVA et al., 2016) é uma métrica para avaliar a qualidade de serviço de deposições de RSUs em redes veículares (VANETs) a partir de seu tráfico veicular. Ele disponibiliza dois parâmetros para o arquiteto da VANET atingir seus objetivos de projeto: o tempo de intercontato $\tau$ e cobertura percentual mínima exigida $\rho$. Um veículo é considerado coberto pela VANET caso não permaneça mais que $\tau$ segundos sem cruzar com alguma RSU. O parâmetro $\rho$ fixa a fração mínima de veículos a se cobrir. Embora $\Gamma_{D}\left(\begin{array}{c}\tau \\ \rho\end{array}\right)$ tenha sido proposta como uma métrica, sua aplicação para minimizar a deposição de RSUs define o Problema da Deposição Gamma (PDG). Silva et al. (2016) propuseram uma formulação de programação linear inteira e uma heurística determinística para o PDG. Faraj et al. (2017) propõem um algoritmo memético para o PDG. Neste trabalho, prova-se que o PDG é NP-Completo, o que era apenas uma conjectura.

\section{O Problema da Deposição Gamma}

Seja um passeio $T_{i}$ uma sequência de vértices tal que haja aresta entre cada par de vértices consecutivos. Seja $L\left(T_{i}\right)$ o número de vértices (distintos ou não) em $T_{i}$ e seja $T_{i}[j]$ o vértice na posição $j$ de $T_{i}, j \in\left\{1, \ldots, L\left(T_{i}\right)\right\}$. Considere-se a operação de subtrair um conjunto $R$ de um passeio $T_{i}$ como resultando em uma coleção de subpasseios de $T_{i}$, a qual é obtida pela remoção em $T_{i}$ de todos os elementos em $R$. Essa operação será denotada por $T_{i} \backslash R$, como no exemplo: $(1,7,2,3,1,4,5,6,7) \backslash\{1,5\}=\{(7,2,3),(4),(6,7)\}$.

Definição 1 (PDG). Seja $G=(V, E)$ um grafo e $T=\left\{T_{1}, \ldots, T_{p}\right\}$ uma coleção de passeios em $G$. Seja $F: T \times \mathbb{Z}_{+}^{*} \rightarrow \mathbb{R}_{+}^{*}$ uma função associando um número positivo a cada etapa $j$ de cada passeio $T_{i} \in T, j \in\left\{1, \ldots, L\left(T_{i}\right)\right\}$. Seja $\tau \in \mathbb{R}_{+}^{*}, \rho \in[0 \%, 100 \%] e$ $K \in \mathbb{Z}_{+}^{*}$ parâmetros adicionais ao problema. $O$ PDG consiste em determinar se existe ou não um conjunto $R \subseteq V$ tal que $|R| \leq K$ e, para pelo menos uma fração $\rho$ dos passeios $T_{i} \in T$, é satisfeita a desigualdade $\sum_{c=1}^{L(C)} F(C, c)<\tau$ para todo $C \in T_{i} \backslash R$. 
Para certificar a corretude de uma instância SIM, deve-se verificar se cada $C \in$ $T_{i} \backslash R$ satisfaz $\sum_{c=1}^{L(C)} F(C, c)<\tau$, o que pode ser executado em tempo polinomial, uma vez que $\left|T_{i} \backslash R\right|<L\left(T_{i}\right)$ Assim, fica claro que o PDG pertence à classe NP. Silva et al. (2016) trataram da situação em que $G$ possuía topologia de grade e propuseram uma discretização simples para expressar qualquer rede rodoviária como grade. Por isso, este trabalho dá um enfoque especial ao Problema da Deposição Gamma em Grades (PDGG).

\section{As Demonstrações}

A partir do Problema da Cobertura de Arestas por Vértices em Grafos Planares com Grau Máximo 3 (PCAVGPGM3), que é NP-Completo (GAREY; JOHNSON, 1977), será feita uma redução polinomial ao Problema da Cobertura de Caminhos por Vértices em Grades (PCCVG), não definido na literatura. Dele, faz-se uma redução polinomial para o PDGG. Seguem-se definições para os problemas intermediários utilizados nas demonstrações.

Definição 2 (PCAVGPGM3). Dado um grafo plano $G=(V, E)$, no qual todos os vértices tem grau menor ou igual a 3, e um número inteiro positivo $K$, existe um conjunto $R \subseteq V$ tal que todas as arestas em E têm pelo menos uma extremidade em $R$ e $|R| \leq K$ ?

Definição 3 (PCCVG). Dada uma grade $G=(V, E)$, uma coleção $S$ de caminhos simples em $G$ e um número inteiro positivo $K$, existe um conjunto $R \subseteq V$ tal que todo caminho $P \in S$ possua ao menos um vértice de $R$ e $|R| \leq K$ ?

\section{Lema 1. O PCCVG é NP-Completo.}

Demonstração. Dado um grafo plano $G=(V, E)$ com grau máximo menor ou igual a 3 e com $K \in \mathbb{Z}_{+}^{*}$, busca-se construir uma grade $G^{\prime}$, uma coleção de caminhos $S$ e um número $K^{\prime} \in \mathbb{Z}_{+}^{*}$. Deve haver uma cobertura $R$ de arestas por vértices em $G$ com $|R| \leq K$ se, e somente se, houver uma cobertura $R^{\prime}$ dos caminhos em $S$ por vértices, no grafo $G^{\prime}=\left(V^{\prime}, E^{\prime}\right)$, com $\left|R^{\prime}\right| \leq K^{\prime}$. Seja $V=\{1, \ldots, n\}$ e $|E|=m$.

Em uma Incorporação em Livro de um grafo $G=(V, E)$, cada vértice é posto linearmente na espinha do livro seguindo a ordem dada por $F_{V}: V \rightarrow \mathbb{Z}_{+}^{*}$ e cada aresta é atribuída a uma de suas páginas por $F_{E}: E \rightarrow \mathbb{Z}_{+}^{*}$. Cada página é um semiplano que parte da espinha do livro. As arestas atribuídas a uma página estão nela contidas e não se tocam. Heath (1985) mostrou como incorporar qualquer grafo plano com grau máximo menor ou igual a $3 \mathrm{em}$ um livro de 2 páginas polinomialmente. Na primeira etapa da nossa transformação, incorpora-se $G$ em um livro de 2 páginas, como exemplifica a Figura 1.

Seja $G^{\prime}=\left(V^{\prime}, E^{\prime}\right)$ uma grade com $V^{\prime}=\{1, \ldots, 3 n\} \times\{1, \ldots, 2 n-1\}$ e $E^{\prime}=$ $\left\{((a, b),(c, d)), \forall(a, b),(c, d) \in V^{\prime}:(|a-c|=1 \wedge b=d) \vee(a=c \wedge|b-d|=1)\right\}$. Seja $H=$ $\left\{\left(3\left(F_{V}(v)\right)-1, n\right), \forall v \in V(G)\right\} \subset V^{\prime}$. Seja $S=\left\{S_{1}, \ldots, S_{m}\right\}$ uma coleção com $m$ caminhos, cada um deles subgrafo de $G^{\prime}$, correspondente a uma das arestas em $E(G)$ e com vértices extremos contidos em $H$. Cada caminho de $(a, b) \in H$ para $(c, d) \in H$ é construído segundo as regras seguintes. Regra (1): Cada caminho tem uma componente vertical. Se a aresta $e \in E(G)$ correspondente for tal que $F_{E}(e)$ é a página esquerda, essa componente passa por vértices na coluna $n-\frac{|b-d|}{3}$. Caso contrário, na coluna $n+\frac{|b-d|}{3}$. Regra (2): Se uma página de $v \in V(G)$ contiver 1 aresta, o caminho correspondente em $S$ tem componente horizontal iniciada no vértice $\left(3\left(f_{V}(v)\right)-1, n\right)$ que toca a componente vertical feita na regra (1). Regra (3): Se uma página de $v \in V(G)$ contiver 2 ou 3 arestas, cada caminho correspondente deixa o vértice $\left(3\left(f_{V}(v)\right)-1, n\right)$ em uma direção diferente (norte, sul ou horizontal) com base na regra (1), evitando cruzamento. Após passar por um vértice, o caminho prossegue horizontalmente até encontrar a componente feita na regra (1). 


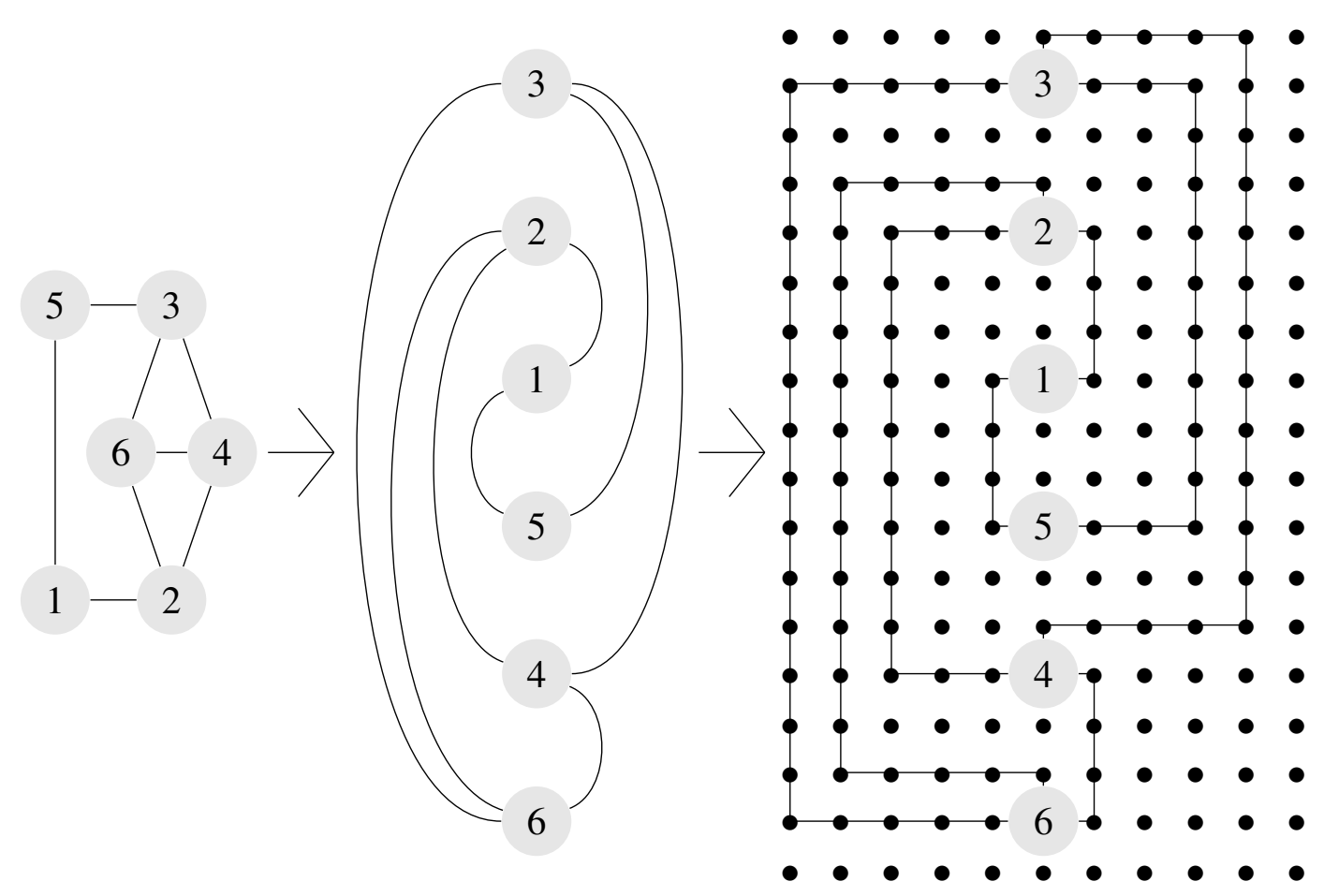

Figura 1. Incorporação de um grafo planar com grau máximo menor ou igual a 3 em um livro de 2 páginas e subsequente transformação em caminhos em grade.

Será provado que $\forall S_{1}, S_{2} \in S, S_{1} \cap S_{2} \subseteq H$. Suponha-se que existam $S_{1}=$ $\left(u_{1}, \ldots, u_{2}\right)$ e $S_{2}=\left(w_{1}, \ldots, w_{2}\right)$ com $S_{1} \cap S_{2} \nsubseteq \amalg$ e, sem perda de generalidade, seja $F_{V}\left(u_{1}\right)<F_{V}\left(u_{2}\right), F_{V}\left(w_{1}\right)<F_{V}\left(w_{2}\right)$ e $F_{V}\left(u_{1}\right) \leq F_{V}\left(w_{1}\right)$. Para simplificar, serão usados os mesmos símbolos $\left(u_{1}, u_{2}, w_{1}\right.$ e $\left.w_{2}\right)$ para se referir aos vértices extremos de $S_{1}$ e $S_{2}$ e aos vértices correspondentes em $V(G)$. Seja o caso em que $S_{1}$ e $S_{2}$ tenham uma extremidade coincidente. Pela regra (3), os subcaminhos horizontais dessa extremidade de $S_{1}$ e $S_{2}$ ocorrem disjuntos nas três linhas em torno da extremidade correspondente, o que é sempre possível devido ao grau máximo de $G$. A regra (1), adicionalmente, garante que não ocorrerá intercepção em qualquer ponto de $S_{1}$ e $S_{2}$ que não pertença a $H$. Considere-se agora o caso em que $u_{1}, u_{2}, w_{1}$ e $w_{2}$ são vértices distintos. São disjuntos os caminhos caso $F_{V}\left(u_{1}\right)$ e $F_{V}\left(u_{2}\right)$ sejam ambos maiores ou menores do que $F_{V}\left(w_{1}\right)$ e $F_{V}\left(w_{2}\right)$. No caso em que $F_{V}\left(u_{1}\right)<F_{V}\left(w_{1}\right)<F_{V}\left(w_{2}\right)<F_{V}\left(u_{2}\right)$, não ocorre intersecção de caminhos devido à regra (1). A única opção restante é $F_{V}\left(u_{1}\right)<F_{V}\left(w_{1}\right)<F_{V}\left(u_{2}\right)<F_{V}\left(w_{2}\right)$. Nesse caso, as arestas $\left(u_{1}, u_{2}\right),\left(w_{1}, w_{2}\right) \in E(G)$ devem certamente pertencer a páginas diferentes na incorporação em livro do grafo, ou seja, $F_{E}\left(u_{1}, u_{2}\right) \neq F_{E}\left(w_{1}, w_{2}\right)$. Logo, $S_{1} \cap S_{2} \subseteq H$.

A Figura 1 exemplifica toda a transformação. É possível encontrar uma cobertura $R^{\prime}$ de caminhos por vértices com $\left|R^{\prime}\right| \leq K^{\prime}=K$ ? No parágrafo seguinte, prova-se por absurdo que a resposta para o PCAVGPGM3 é SIM se, e somente se, também for SIM para o PCCVG em $\left(G^{\prime}, S, K^{\prime}\right)$. O PCCVG está em NP, pois, dado um certificado $R^{\prime}$ para uma instância SIM $\left(G^{\prime}, S, K^{\prime}\right)$, basta checar se $\left|R^{\prime}\right| \leq K^{\prime}$ e se $S_{i} \cap R^{\prime} \neq \emptyset, \forall S_{i} \in S$.

Suponha-se que $\exists R \subseteq V(G)$, com $|R| \leq K$, que é cobertura de arestas por vértices em $(G, K)$ e que a resposta para o PCCVG em $\left(G^{\prime}, S, K^{\prime}\right)$ é NÃO. Como cada vértice de $H$ é associado biunivocamente a um vértice de $V(G)$, é possível obter um conjunto $R^{\prime} \subseteq H$ a partir dos elementos de $H$ associados a $R$. Como cada caminho em $S$ tem as extremidades em $H$ e iguais às extremidades das arestas de $E(G), R^{\prime}$ é uma cobertura para $S$ em $G^{\prime}$ 
e tem-se $\left|R^{\prime}\right| \leq K^{\prime}$, o que contradiz a hipótese. Suponha-se, agora, que $\exists R^{\prime} \subseteq V\left(G^{\prime}\right)$, com $\left|R^{\prime}\right| \leq K^{\prime}$, que é uma cobertura de caminhos por vértices para $\left(G^{\prime}, S, K^{\prime}\right)$ e que a resposta para o PCAVGPGM3 em $(G, K)$ é NÃO. Pela construção proposta, os vértices em $V\left(G^{\prime}\right) \backslash H$ pertencem a, no máximo, um caminho em $S$. Se $\exists S_{i} \in S \operatorname{com} S_{i} \cap\left(R^{\prime} \backslash H\right) \neq \emptyset$, $u \in S_{i} \cap\left(R^{\prime} \backslash H\right)$ cobre exclusivamente $S_{i}$. Logo, pode-se remover $u$ de $R^{\prime}$ e substituí-lo por um vértice em $H \cap S_{i}$. Repetindo isso exaustivamente, obtém-se cobertura de caminhos por vértices $R^{\prime \prime} \subseteq H,\left|R^{\prime \prime}\right|=K^{\prime}$. Assim, pode-se resolver o PCAVGPGM3 com $R \subseteq V(G)$, $|R|=K^{\prime}$, obtendo cada vértice de $V$ associado a $R^{\prime \prime}$, o que contradiz a hipótese.

Teorema 1. O PDG é NP-Completo

Demonstração. Seja $G=(V, E)$ uma grade com $V=\left\{v_{a b}, \forall(a, b) \in\{1, \ldots, m\} \times\{1, \ldots, n\}\right\}$ e $E=\left\{\left(v_{a b}, v_{c d}\right), \forall\left\{v_{a b}, v_{c d}\right\} \subseteq V:(a-c=1 \wedge b=d) \vee(a=c \wedge b-d=1)\right\}$. Seja $S=\left\{S_{1}, \ldots, S_{p}\right\}$ uma coleção de subgrafos de $G$ tal que $S_{i} \in S$ é um caminho em $G$. Com $K \in \mathbb{Z}_{+}^{*}$, pode-se obter uma cobertura de caminhos por vértices $R \subseteq V$ com $|R| \leq K$ ? Dada essa instância do PCCVG, que será referida por $\operatorname{PCCVG}(G, S, K)$, constrói-se a instância $P D G G\left(G^{\prime}\right.$, $\left.T, F, \tau, \rho, K^{\prime}\right)$, do PDGG. Em seguida, mostra-se que $P C C V G(G, S, K)$ é uma instância SIM se, e somente se, $P D G G\left(G^{\prime}, T, F, \tau, \rho, K^{\prime}\right)$ também é. O PDG pertence a NP, pois um certificado $R^{*}$ de SIM pode ser checado em tempo polinomial como mostrado na seção 2 .

Seja $G^{\prime}=G, T=S, K^{\prime}=K, \tau=1$ e $\rho=100 \%$. Para cada passeio $T_{i} \in T, F$ é função definida como $F\left(T_{i}, x\right)=\frac{1}{L\left(T_{i}\right)}, \forall x \in\left\{1, \ldots, L\left(T_{i}\right)\right\}$. Após essa transformação, a seguinte igualdade é válida para cada $T_{i} \in T: \sum_{c=1}^{L\left(T_{i}\right)} F\left(T_{i}, c\right)=1=\tau$ Suponha-se que $\exists R \subseteq V$ que é solução para $P C C V G(G, S, K)$ e não para $P D G G\left(G^{\prime}, T, F, \tau, \rho, K^{\prime}\right)$. Assim, $\exists T_{i} \in T$ contendo subpasseio $C \in T_{i} \backslash R$ que satisfaça $\sum_{c=1}^{L(C)} F(C, c) \geq \tau$. Como $\sum_{c=1}^{L\left(T_{i}\right)} F\left(T_{i}, c\right)=\tau$, pode-se deduzir que $T_{i} \cap R=\emptyset \rightarrow S_{i} \cap R=\emptyset$. Logo, $R$ não resolve $P C C V G(G, S, K)$ já $S_{i}$ não está coberto, o que é uma contradição. Seguindo, suponha-se que $\exists R \subseteq V\left(G^{\prime}\right)$ que é solução para $P D G G\left(G^{\prime}, T, F, \tau, \rho, K^{\prime}\right)$ mas não para $P C C V G(G, S, K)$. Assim, há um caminho $S_{i} \in S, S_{i} \cap R=\emptyset$. Como $T_{i}=S_{i}$, então $T_{i} \cap R=\emptyset$ e $T_{i} \backslash R=\left\{T_{i}\right\}$. Logo, $\exists C \in T_{i} \backslash R$, $\sum_{c=1}^{L(C)} F(C, c)=\tau$, o que contradiz a hipótese de que $R$ resolve o PDGG.

Corolário 1. O PDGG é NP-Completo.

Corolário 2. O PDGG com $\rho=1$ é NP-Completo.

\section{Referências}

FARAJ, M. F.; SARUBBI, J. a. F. M.; SILVA, C. M. da; MARTINS, F. V. C. A hybrid genetic algorithm for deploying RSUs in VANETs based on inter-contact time. In:

Proceedings of the Genetic and Evolutionary Computation Conference Companion. New York, NY, USA: ACM, 2017. (GECCO '17), p. 193-194. ISBN 978-1-4503-4939-0. Disponível em: 〈http://doi.acm.org/10.1145/3067695.3076032〉.

GAREY, M. R.; JOHNSON, D. S. The rectilinear steiner tree problem is NP-complete.

SIAM Journal on Applied Mathematics, SIAM, v. 32, n. 4, p. 826-834, 1977.

HEATH, L. S. Algorithms for embedding graphs in books. Tese (Doutorado) University of North Carolina at Chapel Hill, 1985.

SILVA, C. M.; GUIDONI, D. L.; SOUZA, F. S.; PITANGUI, C. G.; SARUBBI, J. F.; PITSILLIDES, A. Gamma deployment: Designing the communication infrastructure in vehicular networks assuring guarantees on the V2I inter-contact time. In: IEEE. Mobile Ad Hoc and Sensor Systems (MASS), 2016 IEEE 13th International Conference on. [S.1.], 2016. p. 263-271. 